\title{
Quelques éléments remarquables de la faune du Solutréen de Laugerie-Haute (Les-Eyzies-de-Tayac, Dordogne)
}

Some remarkable elements of the Solutrean fauna from Laugerie-Haute (LesEyzies-de-Tayac, Dordogne, France)

Jean-Christophe Castel et Stéphane Madelaine

\section{OpenEdition \\ Journals}

Édition électronique

URL : http://journals.openedition.org/paleo/258

DOI : $10.4000 /$ paleo.258

ISSN : 2101-0420

Éditeur

SAMRA

Édition imprimée

Date de publication : 1 décembre 2006

Pagination : 275-284

ISSN : $1145-3370$

Référence électronique

Jean-Christophe Castel et Stéphane Madelaine, «Quelques éléments remarquables de la faune du Solutréen de Laugerie-Haute (Les-Eyzies-de-Tayac, Dordogne) », PALEO [En ligne], 18 | 2006, mis en ligne le 23 avril 2009, consulté le 07 juillet 2020. URL : http://journals.openedition.org/paleo/258 ; DOI : https://doi.org/10.4000/paleo.258

Ce document a été généré automatiquement le 7 juillet 2020

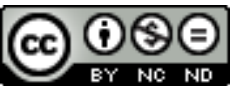

PALEO est mis à disposition selon les termes de la licence Creative Commons Attribution - Pas d'Utilisation Commerciale - Pas de Modification 4.0 International. 


\section{Quelques éléments remarquables de la faune du Solutréen de Laugerie- Haute (Les-Eyzies-de-Tayac, Dordogne)}

Some remarkable elements of the Solutrean fauna from Laugerie-Haute (Les-

Eyzies-de-Tayac, Dordogne, France)

Jean-Christophe Castel et Stéphane Madelaine

\section{NOTE DE L'ÉDITEUR}

Crédits photographiques :

Philippe Jugie du Musée national de Préhistoire (sauf 2-2c, J.-C. Castel). Infographie de Florence Marteau et traductions de Bernard Landry, du Muséum d'histoire naturelle de Genève

Nous tenons à remercier Aline Averbouh pour ses judicieuses remarques.

\section{1 - La longue histoire d'une collection majeure}

Site marquant s'il en est du paysage archéologique du sud-ouest de la France, LaugerieHaute a participé à l'avènement de la Préhistoire. Les fouilles ont vraiment débuté sous l'impulsion de E.Lartet en 1862. Sans revenir sur la longue liste des fouilleurs ${ }^{1}$, retenons l'intervention de ceux qui ont le plus contribué à la constitution des collections paléontologiques : D. Peyrony de 1921 à 1935 (Peyrony et Peyrony 1938), puis F. Bordes dès la fin des années 1950 (Bordes 1958, 1978). Une intervention ultérieure, plus limitée, celle de G. Guichard dans les années 1970 (Guichard et al. 1984), reste à exploiter. 
2 Les données fauniques ont été exploitées par plusieurs chercheurs. Certaines recherches concernent des éléments limités comme les dents dans le cadre d'analyses cémento-chronologiques (Gordon 1988; Burke 1995); d'autres portent sur une large part des collections : J. Bouchud (1966) a travaillé sur les vestiges de renne provenant des fouilles Peyrony puis F. Delpech $(1975,1983)$ a étudié la faune issue des fouilles Bordes.

3 Enfin, à son arrivée au Musée national de Préhistoire des Eyzies et à l'occasion du changement de direction, $\mathrm{S}$. Madelaine procède à un inventaire complet des collections Peyrony dont il publie la partie la plus significative (Madelaine 1989). Nous ajoutons ici le décompte des restes de mammifères pour le Solutréen de Laugerie-Haute Est, fouilles D. Peyrony (tab.1). Parmi les vestiges fauniques des collections Peyrony, des centaines d'os sinon plus ne sont plus rapportés aux secteurs ouest et est du gisement et d'autres sont sans distinction de niveau. A signaler que 1011 vestiges de Laugerie-Haute Est sont désormais notés "Solutréen probable " faute de renseignements fiables.

Tableau 1 - Laugerie-Haute Est et Ouest, Solutréen - Distribution des vestiges de mammifères sauf mammouth - par ensemble culturel.

Table 1 - Laugerie-Haute Est and Ouest, Solutrean - Frequencies of mammal remains - except mammuth on main cultural levels.

\begin{tabular}{|c|c|c|c|c|c|c|c|}
\hline \multirow[b]{2}{*}{ Fouilles } & \multicolumn{2}{|c|}{ Laugerie-Haute Ouest } & \multirow{2}{*}{\multicolumn{2}{|c|}{$\begin{array}{c}\text { Laugerie-Haute Est } \\
\text { et Ouest }\end{array}$}} & \multicolumn{3}{|c|}{ Laugerie-Haute Est } \\
\hline & Bordes & Peyrony & & & & & Peyrony \\
\hline & Delpech & $\begin{array}{l}\text { Madelaine } \\
1989\end{array}$ & $\begin{array}{r}\text { Bouch } \\
\text { dentstalus }\end{array}$ & & Del & 1983 & Madelaine \\
\hline $\begin{array}{l}\text { Solutréen supérieur } \\
\text { et final }\end{array}$ & 1405 & 0 & 226 & 21,9 & 209 & 25,0 & 32 \\
\hline Solutréen moyen & 462 & 937 & 160 & 15,5 & 272 & 32,6 & \\
\hline $\begin{array}{l}\text { Solutréen inférieur } \\
\text { Solutréen sans précision }\end{array}$ & 1663 & $\begin{array}{c}0 \\
239\end{array}$ & 646 & 62,6 & 354 & 42,4 & $\begin{array}{l}13 \\
49\end{array}$ \\
\hline Total & 3530 & 1176 & 1032 & 100 & 835 & 100 & 94 \\
\hline
\end{tabular}

Le matériel attribué au Magdalénien et au Solutréen (fouilles Peyrony et fouilles Bordes) est revu en 2002 par J.-C. Castel dans le cadre de la réalisation de la nouvelle muséographie. Concernant la collection Peyrony, les différences entre les observations de 1989 et celles de 2002/2006 sont infimes (quelques dents séparées de leurs séries ou fragmentées). Pour le matériel de Laugerie-Haute Ouest provenant des fouilles Bordes, la situation est tout à fait semblable et les boîtes créées par F. Delpech dans les années 1970 ont réussi à nous parvenir sans modifications (tab. 1). Il importe de noter que la plupart du matériel récolté par D. Peyrony est désormais enregistré comme Solutréen moyen, ce qui n'était pas le cas lors des examens de Bouchud. La répartition actuelle n'est donc absolument plus conforme à celle observée à l'époque et perd donc sa fiabilité.

Dans ce contexte, il n'est pas raisonnable de tenter de caractériser les différentes phases du Solutréen de Laugerie-Haute Est. Toute tentative doit être précédée d'une reconstitution de l'historique de cette collection. Malgré ces problèmes de changements d'attribution, nous avons considéré que le matériel qui a manifestement changé d'attribution de phase du Solutréen, n'avait toutefois pas circulé entre les différentes grandes phases culturelles. Pour les analyses qui suivent, nous avons toujours considéré les études issues des décomptes des chercheurs de la deuxième 
moitié du XXème siècle (Bouchud et Delpech) comme plus fiables que celles que nous pourrions réaliser à partir des décomptes actuels.

6 Au problème de mélanges de niveaux, s'ajoute celui de la conservation des vestiges après la fouille. Jusqu'à présent les vestiges sont conservés dans des sacs ou dans des boites qui ne dépassent pas 1 kilogramme mais qui peuvent contenir une cinquantaine de vestiges. La moindre manipulation provoque ainsi une usure du matériel.

7 Le matériel dégagé par F. Bordes dans les années 1960 ne semble pas avoir beaucoup souffert. Celui issu des fouilles D. Peyrony est, en revanche, beaucoup moins bien préservé. Les vestiges osseux sont affectés d'une usure de leurs angles et des parties proéminentes de leurs surfaces tout à fait caractéristique de l'érosion d'un matériel sec dans un tiroir qui affecte tant de collections anciennes. La dessiccation provoque de nombreuses cassures. Les dents les plus fragiles finissent par éclater complètement. S'y ajoute l'imprégnation d'une poussière noirâtre parfois assez épaisse qu'il faut ôter avant toute observation approfondie.

8 Mentionnons enfin que, pour les deux collections, la sélection des vestiges lors de la fouille était centrée sur les parties anatomiques identifiables. Les diaphyses n'ont été récoltées que si elles portaient des traces de découpe ou d'utilisation manifestes ou si elles appartenaient à des espèces peu communes. Les os brûlés conservés sont de gros fragments qui ne sont pas forcément représentatifs de ce qui avait été conservé.

\section{2 - Observations archéozoologiques sur le Solutréen de Laugerie-Haute}

9 La plupart des vestiges osseux se rapportent au renne. Les os de cheval sont peu fréquents mais les dents de cette espèce sont relativement abondantes. Bien que le renne soit représenté par sensiblement plus de vestiges (Delpech 1983, tableaux 16 et 17), on peut penser que le cheval jouait vraisemblablement un rôle important dans l'alimentation des solutréens de Laugerie-Haute. Bouquetin, saïga, bovinés et cerf ne sont pratiquement représentés que par des dents. Elles se rapportent à un ou deux individus. La récolte et la conservation de ces vestiges remarquables pour les paléontologues du début du XXème siècle ont pu être plus soignées que celles des innombrables vestiges de renne. Leur rôle dans l'alimentation ne peut donc être déduit du simple rapport de fréquence des différentes espèces. La question de leur chasse dans les alentours de Laugerie peut être posée.

10 En dehors des altérations postérieures à la fouille, le matériel est bien préservé avec des surfaces osseuses bien lisibles, peu affectées de fissurations ou de traces de racines. Cette faible altération peut s'observer de façon homogène sur l'ensemble des petits os sélectionnés pour leurs particularités anatomiques ${ }^{2}$. Cela permet d'avancer qu'il ne s'agit sans doute pas d'un choix de pièces bien conservées par les anciens fouilleurs. La bonne conservation des quelques os brûlés préservés est elle aussi à souligner.

11 Nous n'avons pas examiné les ramures. Des travaux récents ont montré qu'une part importante des bois débités sont des bois de chute qui relèvent par conséquent d'une acquisition distincte de l'introduction de carcasses de rennes (Agoudjil 2005). Les dents fournissent de précieuses indications sur les âges et les saisons d'abattage. Les différentes collections ont sans doute trop souffert (destruction des dents les plus fragiles) pour permettre de rediscuter des observations de J. Bouchud sur les âges 
d'abattage et le sexe des individus (1966). Une analyse des classes d'âge et de la saisonnalité d'abattage demeure possible mais, pour le renne, il y aura une surreprésentation artificielle des individus adultes, les dents des jeunes ayant certainement plus souffert des manipulations de la collection que ces dernières.

Nous avons pu observer de nombreuses stries de désarticulation sur les os; compte tenu de l'absence de diaphyses, les stries de décarnisation sont beaucoup plus rares. Ces observations ne peuvent avoir de portée générale sur les modalités et les objectifs de la découpe. On retiendra que les os du basipode sont assez fréquemment striés. Cette information est cependant triviale dans la mesure où il $\mathrm{y} a \mathrm{eu}$ une forte sélection des os. Dans certains niveaux solutréens, les phalanges fracturées intentionnellement sont nombreuses alors qu'elles sont absentes dans d'autres. Malheureusement, cette différence apparente de récupération de la moelle peut être le résultat de conditions de récolte différentes. D'après l'examen des extrémités des os longs, la fracturation des diaphyses semble standardisée, mais ne faut-il pas, là aussi, rechercher une cause liée à la fouille? Quelques retouchoirs sur diaphyses ont également été isolés lors des fouilles.

\section{3 - Éléments remarquables}

\section{1 - Mammouth : défenses, molaires et os longs}

13 Le site de Laugerie-Haute est bien connu pour avoir livré de nombreux vestiges de mammouth. Il s'agit de larges fragments de défenses mais aussi d'une cinquantaine de fragments plus ou moins importants de dents jugales. Un inventaire précis nécessiterait une révision exhaustive de tous les vestiges conservés dans les différents niveaux et dans les vitrines, ce que nous n'avons pas entrepris. D. Peyrony et E. Peyrony (1938) mentionnent la présence d'une lame d'ivoire dans le Solutréen à pointes à face plane, d'une dizaine de molaires dans le Solutréen à grandes feuilles de laurier et d'une lame de molaire dans le Solutréen à pointes à cran.

Dans le cadre de cette étude, nous avons examiné plusieurs fragments de diaphyses de la couche $H^{\prime \prime}$ des fouilles Peyrony (Solutréen à feuilles de laurier). Deux fragments (longueur-largeur-épaisseur: $125-70-25 / 33$ et $120-98-18 / 30 \mathrm{~mm}$ ) présentent une épaisseur corticale supérieure à celle d'un rhinocéros. Le plus grand de ces fragments correspond probablement à une ulna ou à un tibia de mammouth. Deux autres fragments (110-43-8/25 mm et 132-65-23 $\mathrm{mm}$ ) peuvent correspondre à du mammouth ou du rhinocéros (compte tenu de l'absence de collection de référence adaptée aux Eyzies, l'examen a été réalisé par J.-C. Castel au Muséum d'histoire naturelle de Genève).

Ces fragments présentent une majorité de fractures lisses en spirale caractéristiques d'une percussion lancée sur os frais. Les critères descriptifs de la fracturation intentionnelle sur os frais (cf. Villa et Mahieu 1991) sont valables pour les mammifères de taille inférieure ou égale au bison. La présence de fractures en spirale sur des os de mammouth, ne veut pas forcément dire percussion par l'homme car elles peuvent être confondues avec celles produites lors du piétinement d'un os par d'autres mammouths (ex. Haynes 1988).

16 Aucune épiphyse n'a été retrouvée. S'ils avaient été présents, il est fort probable que de tels vestiges se seraient conservés dans le sol de Laugerie et qu'ils auraient été isolés lors des fouilles anciennes. Il est donc peu vraisemblable qu'il s'agisse de fragments 
provenant d'os entiers introduits dans le gisement. Ces morceaux de diaphyses ont alors sans doute été introduits dans le gisement sous cette forme. Tous sont relativement abîmés et leurs surfaces corticales parfois difficiles à interpréter. L'un des fragments attribués à un très grand mammifère sans précision porte une plage relativement bien délimitée de stigmates très altérés (recoupés par des traces de racines) et de directions désordonnées. Ce n'est pas un retouchoir au sens strict et nous ne sommes pas certains que l'origine de cette plage soit due à une action anthropique.

\section{2 - Ovibos}

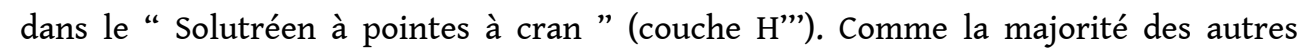
vestiges récoltés, il est probable qu'elle provienne de Laugerie-Haute Ouest, puisque “ ce niveau est particulièrement puissant du côté Ouest ", " du côté Est, il a fourni peu de pièces" (Peyrony et Peyrony 1938, p 42).

Cette dent (une première ou deuxième molaire supérieure gauche) (fig. 1a) correspond vraisemblablement à celle trouvée lors de l'inventaire de 1989 (Madelaine 1989) ; elle est donc attribuable au Solutréen supérieur et confirme le phénomène regrettable de taphonomie de laboratoire qui se serait produit entre 1966 (observations J. Bouchud) et 1989 puisque à l'instar des autres ossements, elle se trouvait dans le Solutréen moyen.

Une autre dent, qui n'avait pas été vue lors de l'inventaire des réserves car faisant partie de la présentation muséographique de l'époque J.Guichard, fut déterminée comme Ovibos après 1989 ; elle est publiée ici pour la première fois. Il s'agit d'une troisième molaire supérieure gauche (fig. 1), marquée “ L.H.O sol.f.l " (marquage Peyrony) ; elle a donc réellement été récoltée dans le Solutréen moyen. Elle n'a pas été publiée en tant que telle par D. et E. Peyrony (ayant pu être attribuée à tort à du cerf ou à du boviné).

Figure 1 - Laugerie-Haute, collection Peyrony, Solutréen. Ovibos moschatus, 1a - première ou seconde molaire supérieure droite ; $1 \mathrm{~b}$ - troisième molaire supérieure droite (Laugerie-Haute Ouest) (Photos Ph. Jugie, MNP). Echelle : $1 \mathrm{~cm}$.

Figure 1 - Laugerie-Haute, Peyrony's collections, Solutrean. Ovibos moschatus, 1a - first or second upper right molar ; $1 b$ - third upper right molar (Laugerie-Haute Ouest) (Photos Ph. Jugie, MNP). Scale : $1 \mathrm{~cm}$.

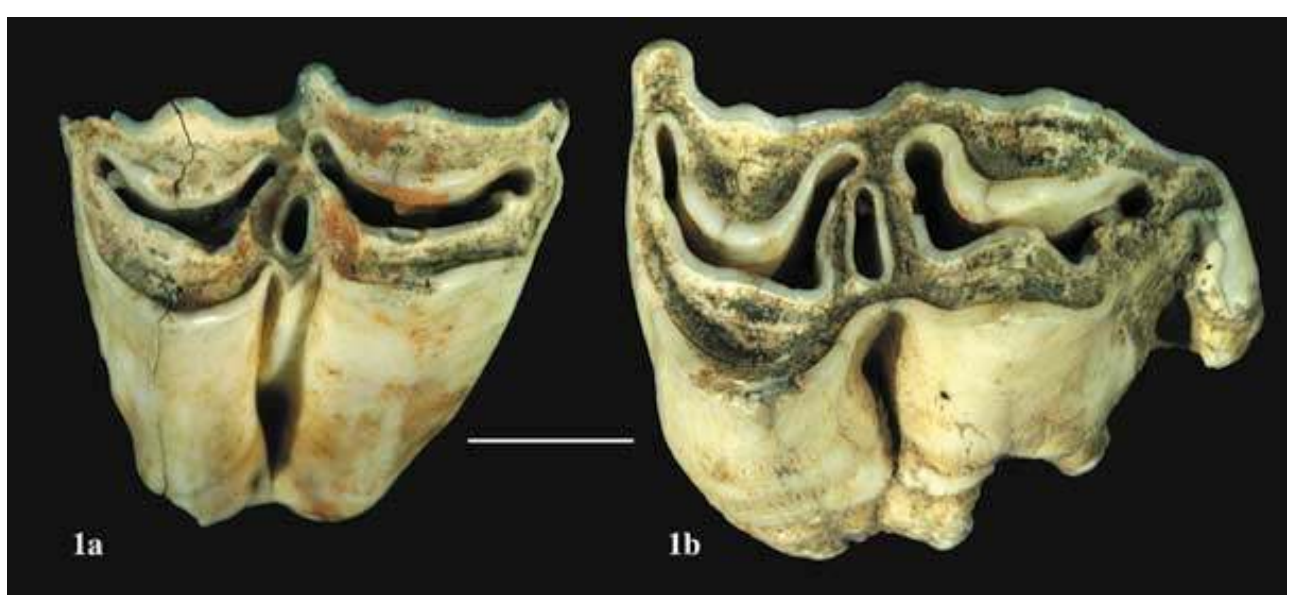

En plus de quelques caractères particuliers déjà décrits (Guérin et Patou-Mathis 1996), la détermination a pu être facilitée et complétée par la comparaison avec un crâne 
actuel d'Ovibos appartenant au Musée national de Préhistoire. Les caractéristiques principales de ces dents sont les suivantes:

- couronne haute (hypsodonte), mais inférieure à celle des bovinés (pour une usure

équivalente);

- présence d'un îlot d'émail central très net sur la face occlusale ;

- médifossettes importantes ;

- lobe mésial anguleux sur la face linguale ;

- lobe distal non anguleux, peu arrondi, avec une crête marquée le long de la couronne entre la face linguale et la face distale pour la 1ère ou 2ème molaire ;

- cette même dent possède une colonnette interlobaire aiguë, développée sur toute la hauteur de la couronne, alors que la 3ème molaire en est dépourvue. Contrairement à ce qui a parfois été écrit (Guérin et Patou-Mathis 1996), cette colonnette n'est pas forcément absente : la preuve en est donnée par le crâne de référence sur lequel les M1 et M3 n'en possèdent pas alors que la $\mathrm{M} 2 \mathrm{en}$ a une. Ce constat nous laisse penser que notre exemplaire fossile pourrait être une M2 ;

- le style distal (métastyle) de la M3 est dilaté transversalement, ébauchant un semblant de troisième lobe (à la manière de l'hypoconulide des troisièmes molaires inférieures ).

21 Il est utile de préciser que ces deux dents, au vu de leur usure différentielle, appartiennent à deux individus différents, ce qui est logique étant donné les présomptions vraisemblables de leur appartenance à deux couches différentes. Il est à noter également que la $\mathrm{M}^{1}$ ou 2 présente quelques traces d'ocre sur la couronne et surtout sur la face occlusale ainsi qu'à l'intérieur de la fossette distale.

La présence de ces deux dents revêt un intérêt particulier : ce sont les seuls vestiges de cette espèce dans le Solutréen d'Aquitaine (voire probablement de France) et, à notre connaissance, ce taxon n'a été décrit, par ailleurs, qu'extrêmement rarement :

- un fragment de crâne et deux fragments de métapodes découverts par Galou à Gorge d'Enfer (Harlé 1901) provenant sans doute, d'après la description des lieux , de l'abri du Poisson et donc attribuables soit à l'Aurignacien ancien, soit au Gravettien ;

- des os de pattes découverts par Lartet, également à Gorge d'Enfer, dans une petite grotte (Harlé 1901) ;

- les découvertes d'os de bœuf musqué sont beaucoup plus communes en Europe centrale et du nord (ex. Soergel 1942).

\section{3 - Antilope saïga}

Les restes d'antilope saïga sont absents des décomptes de F. Delpech (1983) pour le Solutréen de Laugerie-Haute Est et Ouest des fouilles de F. Bordes. En revanche, dans le matériel provenant des fouilles de D. Peyrony de Laugerie-Haute Ouest, six vestiges attribuables à cette espèce ont été isolés (Madelaine, 1989)33), dont une $M_{3}$ inférieure et une $\mathrm{M}^{1}$ ou $\mathrm{M}^{2}$ supérieure tout à fait caractéristiques ayant fait l'objet précédemment d'une erreur de détermination (elles sont effectivement marquées “ LH. Sol., C. ibex ").

La présence de l'antilope saïga dans le Solutréen supérieur du sud-ouest de la France est désormais un fait reconnu (cf. Dujardin et Timula 2005). Dans les sites de la moitié nord de l'Aquitaine (Le Placard, Fourneau-du-Diable et Combe-Saunière), elle est représentée par des nombres de restes relativement importants. Dans les sites du sud du Périgord et dans le Quercy elle est très rare (un unique reste aux Jamblancs comme 
au Pech-de-la-Boissière). Ces vestiges isolés ne constituent pas des preuves suffisantes pour attester d'une chasse locale (Castel et al. 2005).

\section{4 - Retouchoirs sur dents de cheval}

Les retouchoirs sur dents jugales de cheval sont connus depuis l'identification faite par H. Rutot en 1908 dans la caverne d'Hastière en Belgique. Pourtant il faut attendre leur redécouverte par D. Armand et A. Delagnes en 1998 pour qu'ils suscitent à nouveau l'intérêt. Depuis, d'autres exemplaires ont été trouvés à la Ferrassie (Savignac-deMiremont) et au Pigeonnier (Gensac) (Castel et al. 2003). Toutes ces pièces sont attribuées à l'Aurignacien et au Moustérien.

Lors de l'examen archéozoologique des collections solutréennes de Laugerie-Haute, deux exemplaires caractéristiques ont été identifiés. D'autres exemplaires ont pu nous échapper, masqués par la poussière qui couvre les stigmates. Pour s'assurer du nombre exact de ces pièces à Laugerie-Haute, il sera nécessaire de nettoyer l'intégralité des dents.

Troisième molaire inférieure droite - Laugerie-Haute Ouest, fouilles D. Peyrony, couche $H^{\prime \prime}$, Solutréen à feuilles de laurier (fig. 2-1)

Cette dent est relativement bien conservée mais quelques fragments d'émail ont disparu depuis sa découverte. Sa face mésiale (antérieure) $(\mathrm{L}=64 \mathrm{~mm})$ a fait l'objet d'un intense raclage préparatoire dans le sens vertical, ou axe longitudinal, qui a entraîné une suppression de matière de 1 à $2 \mathrm{~mm}$ d'épaisseur dans la partie moyenne. Ces stries de raclage sub-parallèles sont présentes pratiquement jusqu'à la bordure occlusale le long du parastylide. En revanche, il n'est pas certain que ce raclage descende jusqu'à la base de la racine. Il déborde du parastylide pour affecter marginalement l'extension mésiale du métaconide. Cela a provoqué la disparition du cément et de l'émail sur une partie de la hauteur. La dentine, ou ivoire, apparaît dans la partie centrale. Les angles entre la face antérieure et les faces vestibulaire et linguale sont rendus plus vifs par cet enlèvement de matière.

Les stigmates d'impacts sont répartis en deux zones proches des extrémités comme cela s'observe fréquemment pour les retouchoirs sur diaphyses (ex. : Auguste 2002; Castel 1999 ; Malherba et Giacobini 2002 ; Vincent 1993). Dans la zone supérieure, le nombre d'impacts dépasse la cinquantaine; dans la zone inférieure il est situé entre 30 et 50. Les entailles de formes allongées, parfois cunéiformes, dominent; par endroits, elles sont plus irrégulières et à peu près circulaires (près de l'extrémité occlusale). Les arrachements cunéiformes les plus caractéristiques sont conservés sur l'ivoire. Les marques sur l'émail sont moins profondes et moins longues parce que cette matière est plus résistante. La direction des entailles forme un angle de 65 à $75^{\circ}$ par rapport à l'axe de la pièce (allongement de la dent) comme cela s'observe sur de nombreux retouchoirs. L'orientation des stigmates est identique pour les deux zones. 
Figure 2 - 1, Laugerie-Haute Ouest, collection Peyrony, Solutréen. Equus caballus, troisième molaire inférieure droite ; $1 \mathrm{a}$ - face vestibulaire, 1b, c, d- détails de la face mésiale (antérieure) utilisée. Echelle $1 \mathrm{~cm}$.

Figure 2 - 2, Laugerie-Haute Ouest, collection Peyrony, Solutréen. Equus caballus, première ou seconde molaire inférieure droite ; $2 a$ - face vestibulaire, $2 b, c$ - détails de la face distale utilisée, $2 \mathrm{~d}-$ face linguale. Echelle $1 \mathrm{~cm}$.

Figure 2 - 1, Laugerie-Haute Ouest, Peyrony's collection, Solutrean. Equus caballus, third right lower molar ; $1 \mathrm{a}$ - external surface, $1 \mathrm{~b}, \mathrm{c}, \boldsymbol{d}$ - close-up of the utilized zones on anterior surface. Scale $1 \mathrm{~cm}$. Figure 2 - 2, Laugerie-Haute Ouest, collection Peyrony, Solutréen. Equus caballus, first or second right lower molar ; $2 a$ - external surface, $2 b, c$ - close-up of the utilized zone on back surface, $2 d$ - internal view. Scale $1 \mathrm{~cm}$.

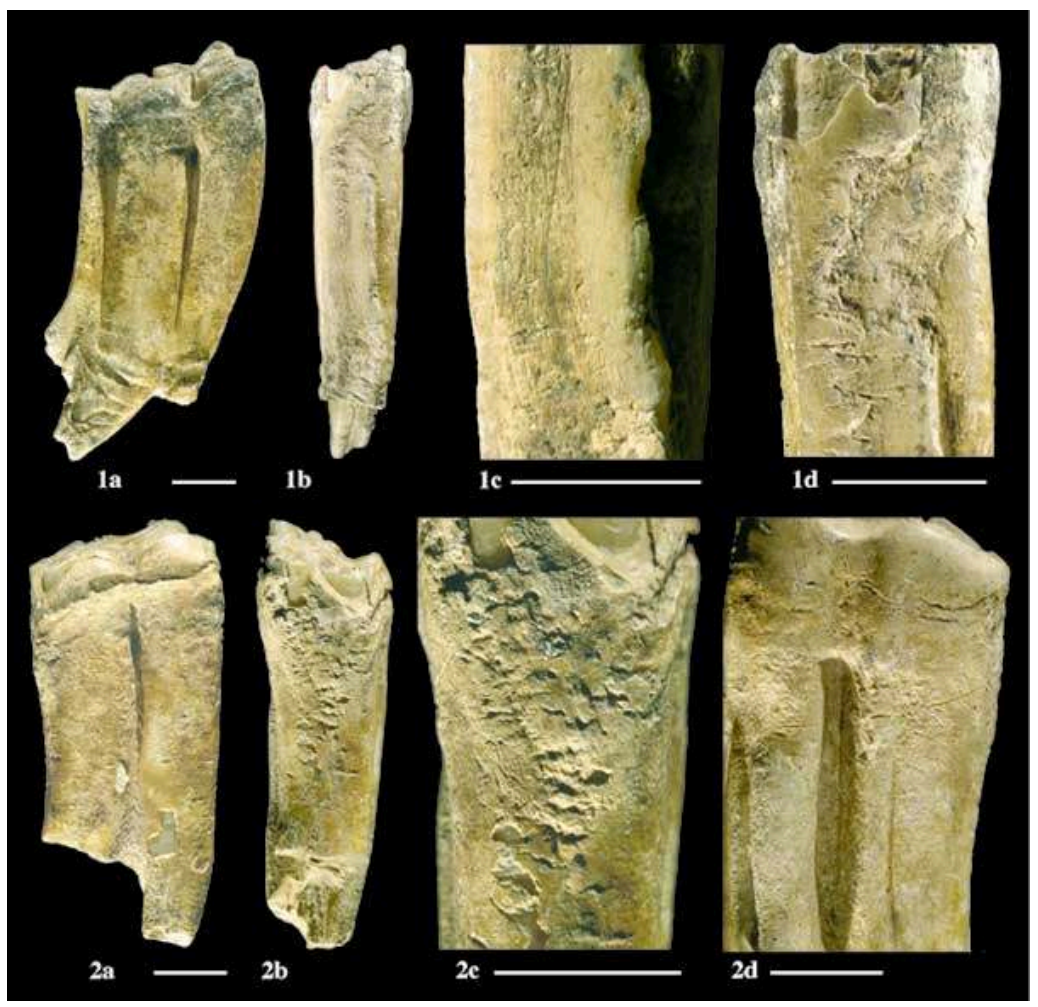

Première ou seconde molaire inférieure droite - Laugerie-Haute Ouest, fouilles Peyrony, Solutréen sans précision (fig. 2-2)

Cette dent a souffert de son mode de stockage depuis sa découverte. Elle montre toutefois une série de stigmates sub-parallèles sur sa face distale de même direction que ceux observés pour la dent précédente. Le nombre d'impacts peut être estimé entre 30 et 50 . On observe quelques stries verticales isolées qui ne ressemblent pas aux stries de raclages telles qu'elles peuvent s'observer sur la dent précédente ou sur les diaphyses. Signalons que la face linguale porte quelques stries de direction mésiodistale qui ne sont pas forcément en relation avec l'utilisation technique de la dent (fig. 2-2d).

30 Le mode d'utilisation de ces deux pièces est tout à fait semblable (impacts provoquant des stigmates cunéiformes) mais on peut observer une préparation et une utilisation beaucoup plus importante pour la $\mathrm{M}_{3}$. Bien que la surface de contact et la prise en main soient beaucoup plus réduites que ce qu'offrent les diaphyses d'herbivores (bovinés, cheval et renne), le mode d'utilisation de ces dents semble similaire.

31 Il est possible qu'un nombre significatif de retouchoirs sur dents jugales d'équidés ait pu échapper à la sagacité des archéozoologues et plus encore à celle des 
paléontologues. Après l'identification de ce type de vestiges dans le Paléolithique moyen et l'Aurignacien, cette identification dans le Solutréen de Laugerie-Haute permet de s'interroger sur leur existence dans d'autres sites solutréens et dans d'autres cultures du Paléolithique supérieur.

A noter que toutes les dents de chevaux utilisées identifiées à ce jour sont des dents jugales inférieures, mais il s'agit peut-être d'un effet d'échantillonnage. Pour l'instant nous ne connaissons pas de dents de bovinés utilisées, mais la présence de nombreux stigmates sur l'émail des dents de cheval de Laugerie-Haute permet de suspecter une possible utilisation de ces dents dépourvues de cément dans la partie haute de leurs couronnes. La diversité des matières premières et des supports utilisés pour la réalisation des retouchoirs a déjà été constatée (ex. Patou-Mathis 2002). Elle est susceptible de correspondre à une gamme d'utilisations correspondant à des activités distinctes. Cette découverte réalisée dans le matériel de Laugerie-Haute souligne que si ces vestiges sont encore insuffisamment connus, c'est dans une large mesure parce qu'ils n'ont pas été recherchés.

\section{4 - Discussion et perspectives}

Laugerie-Haute est l'un des sites majeurs qui s'inscrivent dans le débat sur la présence et la caractérisation de sites d'agrégation (Conkey 1980, 1992). Les données paléontologiques du matériel du Solutréen révèlent deux éléments qui sont à verser au dossier: la présence du bœuf musqué qui n'est signalé qu'exceptionnellement en Aquitaine et l'importante récolte de vestiges de mammouth que ce soit l'ivoire des incisives, les dents jugales mais aussi quelques os longs. Ces deux éléments de caractère exceptionnel ne peuvent pas seulement être attribués à un effet de probabilité lié à la grande extension des fouilles. En effet, dans tous les autres sites réunis on ne trouve pas autant d'éléments si remarquables. Peut-on alors considérer ces particularités du Solutréen de Laugerie-Haute comme la manifestation d'un statut particulier de ce site au cours de cette période?

Le nouvel examen des collections de Laugerie-Haute n'a apporté que peu précisions sur les choix et les modes d'exploitation du milieu par les solutréens. Nos observations ne vont guère au-delà de ce qu'avaient apporté celles de J. Bouchud (1966) et de F. Delpech (1983). Les collections ont été constituées selon des objectifs qui ne le permettent guère; de plus, elles ont subi des manipulations et une érosion dans les tiroirs qui rend vaine toute étude approfondie. Seules les analyses cémento-chronologiques nous semblent prometteuses. Même d'extension limitée, les fouilles futures apporteront une information immensément plus riche sur l'exploitation du renne et des autres espèces chassées. Ce n'est qu'ainsi que le statut du bœuf musqué peut espérer être mieux compris ; c'est aussi le cas de toutes les espèces représentées par un tout petit nombre de restes (voir Delpech 1983). 


\section{BIBLIOGRAPHIE}

AGOUDGIL A. 2005 - Essai de caractérisation des industries en matières dures animales

solutréennes : Apport de l'étude du niveau Solutréen moyen (couche H" à feuilles de laurier) de Laugerie-Haute Ouest à la connaissance des modalités de débitage du bois de cervidé. Mémoire de DEA, Université de Paris I - Panthéon-Sorbonne.

ARMAND D. et DELAGNES A. 1998 - Les retouchoirs en os d'Artenac (couche 6c) : perspectives archéozoologiques. In : J.-Ph. Brugal, L. Meignen, M. Patou-Mathis (Ed.), Economie préhistorique : les comportements de subsistance au Paléolithique, XVe Rencontres internationales d'archéologie et d'Histoire d'Antibes, Editions APDCA, Sophia Antipolis, 1998, p. 205-214.

AUGUSTE, P. 2002 - Fiches éclats diaphysaires du Paléolithique moyen : Biache-Saint-Vaast (Pasde-Calais) et Kulna (République Tchèque). In : Patou-Mathis, M. (Dir.) : Industrie de l'os préhistorique. Cahier X : Compresseurs, percuteurs, retouchoirs. Paris : Editions S.P.F., 39-57.

Bordes F. 1958 - Nouvelles fouilles à Laugerie-Haute, premiers résultats. L'Anthropologie, 62-3/4, Paris, 205-244.

Bordes F. 1978 - Le Protomagdalénien de Laugerie-Haute-Est (fouilles F : Bordes), Bulletin de la Société Préhistorique Française, 75-11/12, 501-521.

Bouchud J. 1966 - Essai sur le Renne et la climatologie du Paléolithique moyen et supérieur. Périgueux, Imprimerie Magne.

Burke A. 1995 - Prey Movements and Settlement Patterns During the Upper Palaeolithic in Southwestern France. Oxford, British Archaeological Reports, BAR International Series, 619.

CASTEL J.-C. 1999 - Comportements de subsistance au Solutreén et au Badegoulien d'après les faunes de Combe-Saunière (Dordogne) et du Cuzoul de vers (Lot). Thèse, Université Bordeaux I, non publiée.

Castel J.-C., Chauvière F.-X. et Madelaine S. 2003 - Sur os et sur dents : les “ retouchoirs” aurignaciens de la Ferrassie (Savignac-de-Miremont, Dordogne). Paléo, 15, p.29-50.

Castel J.-C., Chadelle J.-P. et Geneste J.-M. 2005 - Nouvelle approche des territoires solutréens du Sud-Ouest de la France. In : J. Jaubert, M. Barbaza (dir), Territoires, déplacements, mobilité, échanges durant la Préhistoire, 126 e congrès du CTHS, Toulouse, 2001. Paris, Editions du CTHS. p. 279-294.

Conkey M. W. 1980 - The Identification of Prehistoric Hunter-gatherer Aggregation sites : The Case of Altamira. Current Anthropology $21: 609-630$.

Conkey M. W. 1992 - Les sites d'agrégation et la répartition de l'art mobilier, ou : y a t'il des sites d'agrégation magdaléniens ? In : France. Comité des Travaux Historiques et Scientifiques. Section de préhistoire et de protohistoire (1988, Chancelade). Le Peuplement Magdalénien, Paléogéographie physique et Humaine. Paris, CTHS, p. 19-28.

DELPECH F. 1975 - Les faunes du Paléolithique supérieur dans le Sud-Ouest de la France. Thèse de Doctorat es Sciences naturelles, Université de Bordeaux I, non publiée.

DELPECH F. 1983 - Les faunes du Paléolithique Supérieur dans le Sud-Ouest de la France, Paris : Editions du CNRS. Cahiers du Quaternaire, $n^{\circ} 6$. 
Dujardin V. et Timula S. 2005 - Relecture chronologique de sites paléolithiques et épipaléolithiques anciennement fouillés en Poitou-Charentes, Bulletin de la Société Préhistorique Française, 102-4, 771-788.

Gordon B.C. 1988 - Of Men and Reindeer in French Magdalenian Prehistory. Oxford, British Archaeological Reports, BAR International Series 390.

GuÉRIN C. et PATOU-MATHIS M. 1996 - Les grands mammifères plio-pléistocènes d'Europe, Masson, collection Préhistoire, 292 p.; 73 fig.

Guichard G., Delluc, B. et Delluc G. 1984 - Abri de Laugerie-Haute, in : L'Art des Cavernes, 138-143.

HARLE E. 1901 - Un crâne de bœuf musqué, des Eyzies (Dordogne). Bulletin de la Société Géologique de France, 4 ème série, tome I, p. 455.

Haynes G. 1988 - Longitudinal studies of African elephant death and bone deposits. Journal of Archaeological Science, 15, 131-157.

MADELAINE S. 1989 - Contribution des anciennes fouilles à la connaissance des ongulés et de leurs milieux durant le Würm récent en Dordogne. Paléo, 1, 36-46.

Malerba, G et Giacobini, G. 2002 - Fiche éclats diaphysaires avec marques transversales d'utilisation. In : Patou-Mathis, M. (Dir.) : Industrie de l'os préhistorique. Cahier X : Compresseurs, percuteurs, retouchoirs. Paris : Editions S.P.F., 29-37.

Patou-Mathis M. (Dir.) 2002 - Industrie de l'os préhistorique. Cahier X : Compresseurs, percuteurs, retouchoirs. Paris : Editions S.P.F.

Peyrony D. et Peyrony E. 1938 - Laugerie-Haute près des Eyzies (Dordogne), Paris : Masson, Archives de l'Institut de Paléontologie Humaine, 19.

RUTOT A. 1908 - Sur la répartition des os utilisés en Belgique, Bulletin de la Société Préhistorique de France, 11, 280-281.

SOERGEL W. 1942 - Die Verbreitung des diluvialen Moschusochsen in Mitteleuropa. Beitr. z. Geol. von Thüringen, 7, 75-95.

Villa P. et Mahieu E. 1991 - Breakage Patterns of Human long Bones. Journal of Human Evolution 21, 27-48.

VINCENT A. 1993 - L'outillage osseux au Paléolithique moyen : une nouvelle approche. Thèse, Université de Paris $\mathrm{X}$, non publiée.

\section{NOTES}

1. Le Muséum d'histoire naturelle de Genève détient, dans les collections du Département d'archéozoologie, du matériel provenant de fouilles du XIXème siècle. Il s'agit de la collection Gosse qui renferme une vingtaine d'os de renne de Laugerie-Basse et de la collection A. Favre qui renferme une quarantaine d'os de renne notés “Laugerie". Ils ne sont pas attribués culturellement. Il s'agit de fragments plutôt bien conservés provenant de différentes parties du squelette (mandibules, vertèbres, extrémités d'os longs, basipode, phalanges).

2. Cette caractéristique se retrouve sur le matériel conservé à Genève.

3. S. Madelaine (1989) signale quatre restes sur six dans le Solutréen moyen or nous avons vu que les effectifs du Solutréen moyen avaient fortement progressé depuis les premières analyses paléontologiques et que cette attribution à l'intérieur du Solutréen était désormais peu fiable. 


\section{RÉSUMÉS}

Laugerie-Haute est l'un des sites majeurs qui s'inscrivent dans le débat sur la présence et la caractérisation de sites d'agrégation (Conkey 1980, 1992). L'examen des collections de LaugerieHaute dans le cadre de la préparation de la nouvelle muséographie du Musée national de Préhistoire des Eyzies, nous a permis de remettre au jour quelques éléments remarquables de la faune attribuée au Solutréen. Il s'agit de la présence exceptionnelle du bœuf musqué (Ovibos moschatus) signalée dans un nombre très limité de gisements en France et de l'importante récolte de vestiges de mammouth, qu'il s'agisse des vestiges dentaires déjà décrits par Peyrony mais aussi de quelques fragments de diaphyses d'os longs. En outre, nous mentionnons la présence d'antilope saïga (Saiga tatarica) qui est reconnue dans plusieurs sites solutréens du sudouest de la France.

Enfin, deux retouchoirs réalisés sur des dents jugales inférieures de cheval ont été découverts. Ces vestiges, jusqu'ici identifiés seulement dans l'Aurignacien et le Moustérien doivent désormais être recherchés dans l'ensemble du Paléolithique supérieur. Ces éléments de caractère exceptionnel ne peuvent pas seulement être attribués à un effet de probabilité lié à la grande extension des fouilles. En effet, dans tous les autres sites solutréens réunis, on ne trouve pas autant d'éléments aussi remarquables.

Laugerie-Haute is one of the most famous French Upper Paleolithic sites on which the concept of aggregation sites has been developped (Conkey 1980, 1992). The examination of collections from the site during the preparation of the new museography of the National Museum of Prehistory in Les Eyzies allowed us to rediscover some remarkable elements of the Solutrean fauna. We note the presence of the Muskox (Ovibos moschatus), previously known only in few sites. We also note the presence of Mammuth fragments such teeth described before by D. Peyrony, and especially long bone diaphyses. We also mention the presence of the saiga antelope (Saiga tatarica) which is represented in several solutrean sites in the region. Finally, two horses lower teeth modified as “ retouchers " were found. Such objects where until now only recovered in Aurignacian and Mousterian sites and should be searched in all Upper Paleolithic sites.

The presence of these elements is not only due to the large size of the excavations since the middle of the $19^{\text {th }}$ century. Actually, there are no such remarkable pieces in all remaining solutrean sites of the South-West of France.

\section{INDEX}

Keywords : Laugerie-Haute, Solutrean, laboratory taphonomy, Ovibos moschatus, Mammuthus primigenius, Saiga tatarica, retouchers on horse teeth

Mots-clés : Laugerie-Haute, Solutréen, taphonomie de laboratoire, Ovibos moschatus, Mammuthus primigenius, Saiga tatarica, retouchoir sur dent de cheval

\section{AUTEURS}

\section{JEAN-CHRISTOPHE CASTEL}

Département d'archéozoologie, Muséum d'histoire naturelle, Route de Malagnou 1, CP 6434, CH1211 Genève 6, jean-christophe-castel@ville-ge.ch 


\section{STÉPHANE MADELAINE}

Musée national de Préhistoire - 24620 Les Eyzies. Université Bordeaux I - UMR 5199 - PACEA Institut de Préhistoire et de Géologie du Quaternaire, avenue des Facultés - 33405 Talence cedex stephane.madelaine@culture.gouv.fr 